\title{
Chancen und Herausforderungen digitaler Arbeit für die Führung und Zusammenarbeit
}

\author{
Conny Herbert Antoni ${ }^{1}$ Falko von Ameln ${ }^{2}$
}

Online publiziert: 27. Oktober 2017

(c) Springer Fachmedien Wiesbaden GmbH 2017

In diesem Themenheft der Zeitschrift „Gruppe. Interaktion. Organisation. (GIO) “ werden die Auswirkungen der zunehmenden Digitalisierung der Arbeit auf die Führung und Zusammenarbeit beleuchtet. Der Einsatz digitaler Technologien ermöglicht nicht nur die Vernetzung von Maschinen und Anlagen, sondern erlaubt es Unternehmen auch, die Zusammenarbeit räumlich und zeitlich zu flexibilisieren und zu vernetzen. Damit entstehen neue Chancen und Herausforderungen für die Führung und Zusammenarbeit, die in neun Beiträgen aus unterschiedlichen Perspektiven beleuchtet werden.

In dem Beitrag Digitalisierung der Arbeit: Konsequenzen für Führung und Zusammenarbeit geben Conny Antoni und Christine Syrek einen Überblick über den aktuellen Forschungsstand zu Merkmalen, Anforderungen und Auswirkungen digitaler Führung und Zusammenarbeit bei komplexen und dynamischen Projektaufgaben. Sie prüfen dabei insbesondere die These, dass es bei komplexer digitaler $\mathrm{Zu}$ sammenarbeit zur Leistung beiträgt, wenn Führungsfunktionen an das Team delegiert werden und personale Führung durch strukturelle Führung, etwa durch Ziel-, Feedback- und Belohnungssysteme, ergänzt wird. Die Autoren zeigen, dass die vorliegenden Befunde für die Annahme dieser These sprechen, sofern in einem Team gemeinsame Vorstellungen zu inhaltlichen und zeitlichen Zielen, Aufgaben, Handlungsstrategien, Rollen und Verantwortlichkeiten bestehen, und leiten daraus Empfehlungen für die Praxis

Prof. Dr. Conny Herbert Antoni

antoni@uni-trier.de

1 FB I, Arbeits-, Betriebs- und Organisationspsychologie, Universität Trier, 54286 Trier, Deutschland

2 Organisationsberatung, Am Diekschloot 11b, 26506 Norden, Deutschland ab. Viele andere Fragen zu den Auswirkungen moderner Informations- und Kommunikationstechnologien auf Führung und Zusammenarbeit und auf die Belastungen und Beanspruchungen der Beschäftigten lassen sich durch die vorliegenden Studien allerdings noch nicht beantworten und eröffnen Perspektiven für künftige Untersuchungen.

Welche Chancen und Herausforderungen die AOK in Niedersachsen durch die Digitalisierung der Arbeit sieht und wie die AOK sich darauf einstellt, beschreibt Ulrike Köhler in dem von Christine Syrek geführten Interview $H R$ in der Arbeitswelt 4.0 bei der AOKN. Ihrer Ansicht nach eröffnet die Digitalisierung die Möglichkeit, Prozesse kundenspezifischer zu gestalten und Arbeit etwa durch den Einsatz neuer collaboration tools, durch mobiles Arbeiten und durch Home-Office zu flexibilisieren. Mitarbeiter könnten mehr Eigenverantwortung übernehmen und Entscheidungen selbst treffen. Dies erfordere, dass Führungskräfte die Entscheidungsmechanismen klar festlegen, stärker werteorientiert führen, Mitarbeitern den Sinn von Veränderungsprozessen vermitteln und sie bei ihren Aufgaben begleiten, beraten und coachen sowie als Vorbild für ihre Teams agieren.

Mit der Rolle der operativen Führungskräfte bei digitaler Zusammenarbeit beschäftigt sich der konzeptionelle Beitrag Ressourcenstärkende Führung - operative Führungskräfte in virtuellen Kontexten von Erich Latniak. Diese Beschäftigtengruppe, die an der Schnittstelle von Kunden, Management und Team arbeitet und mit den sich daraus ergebenden widersprüchlichen Anforderungen konfrontiert ist, wurde bisher kaum untersucht, obwohl es Hinweise für spezifische Belastungs- und Beanspruchungsmuster gibt. Auf der Basis ausgewählter offener Forschungsfragen zu virtueller Teamarbeit und Führung sowie deskriptiver Befunde zur Belastungs- und Ressourcensituation operativer Führungskräfte in IT- und Entwicklungsbereichen entwickelt der Autor Forschungsperspektiven zu Anforderungen und 
Bedingungen der Arbeit operativer Führungskräfte bei virtueller Teamarbeit. Dabei werden insbesondere Rahmenbedingungen und technische Voraussetzungen als treibende Faktoren in den Blick genommen.

Mit der Frage, wie die digitale Transformation das Verständnis der Führung in einem Verlag verändert, beschäftigt sich auch der Praxisbeitrag von Joachim Rotzinger Blick in die Praxis: Haufe Gruppe. Am Beispiel der digitalen Transformation der Haufe Gruppe beleuchtet der Artikel exemplarisch erfolgreiche und Erfolg versprechende Herangehensweisen für Geschäftsstrategie und Personalführung. Er zeigt anschaulich, dass digitale Transformation auch soziale Transformation erfordert und wie dies durch Wertschätzung, Offenheit, Transparenz, Vertrauen und vor allen Dingen durch Flexibilität im Führungsstil gelingen kann.

Der Beitrag Virtuelle Arbeitsstrukturen durch Vertrauen, Zeitkompetenz und Prozessfeedback fördern von Frank Müller, Rebekka Mander und Ulrike Hellert beschäftigt sich mit Vertrauen, Zeitkompetenz und Prozessfeedback als zentralen Herausforderungen virtueller Kooperations- und Teamstrukturen. Er verdeutlicht, dass Vertrauen in virtuellen Arbeitsstrukturen auf eine andere Weise entsteht als in Präsenzteams. Die Autor/-innen argumentieren, dass unter diesen Bedingungen Zeitkompetenz für die Entwicklung von Vertrauen besonders wichtig ist, da Teams im virtuellen Umfeld flexibler arbeiten und Teammitglieder mehr Verantwortung und Kontrolle übernehmen als bei vergleichbaren Präsenzteams. Um Zeitkompetenz und Vertrauen zu fördern, empfehlen die Autor/-innen Prozessfeedback und transformationale Führung.

Der Praxisbeitrag Digitalisierung des Talentmanagements bei der Deutschen Bahn von Nils Stegemann beschreibt, wie die Deutsche Bahn ihre Prozesse der Personalauswahl, Personalentwicklung und Stellenbesetzung den Anforderungen der Digitalisierung anpasst. Ziel ist es, schon im Auswahlprozess das Unternehmen von morgen für die Teilnehmer erlebbar zu machen, indem Zusammenarbeit in agilen Settings simuliert wird. In der Personalentwicklung sollen soziale Netzwerke die digitale Vernetzung und selbstgesteuerte Aktivitäten der Talent Community sowie die aktive Zusammenarbeit mit Vertretern des Top Managements zu strategischen Herausforderungen ermöglichen. Ferner soll mittelfristig das Talentmanagement in Richtung Selbstselektion (,Wo möchte ich dabei sein?“) für eher fachlich organisierte Talent Communities of Interests ausgerichtet werden.

Um Team- und Projektarbeit über Standorte hinweg zu vernetzen, Wissen und Daten $\mathrm{zu}$ teilen, Projekte $\mathrm{zu}$ organisieren und $\mathrm{zu}$ unterstützen, werden verstärkt internetbasierte kollaborative Anwendungen eingesetzt. Der Beitrag Gestaltung internetbasierter kollaborativer Team- und Projektarbeit von Stefan Klötzer, Thomas Hardwig und Margarete Boos analysiert anhand eines Gestaltungsmodells für räumlich verteilte kollaborative Arbeit, ob diese Vorteile tatsächlich genutzt werden können. Exemplarisch werden an einem Praxisbeispiel Gestaltungsmöglichkeiten, aber auch Probleme, die bspw. durch die parallele Nutzung von Anwendungen entstehen können, aufgezeigt und im Modell auf den Ebenen Strategie, Methoden und Technik verortet.

In dem Beitrag Informationsüberlastung bei digitaler $\mathrm{Zu}$ sammenarbeit - Ursachen, Folgen und Interventionsmöglichkeiten, der den Themenschwerpunkt abschließt, analysieren Conny Antoni und Thomas Ellwart den aktuellen Stand der Forschung zu Einflussfaktoren und Folgen von Informationsüberlastung bei der medienvermittelten Kommunikation im Team. Die Autoren erörtern, welche Wirkungsmechanismen $\mathrm{zu}$ einer Überlastung führen können und wie Führungskräfte durch Aufgaben- und Teamgestaltung, Schulungsmaßnahmen und Teamreflexion Informationsüberlastung vermeiden und zu einer effizienten IKT vermittelten Kommunikation im Team beitragen können.

Mit seinem Beitrag Change the Change Management! schlägt Hans-Joachim Gergs die Brücke zum offenen Teil des Heftes. In einer digitalisierten Arbeitswelt, so die These des Autors, können Organisationen nur dann überleben und Erfolg haben, wenn sie sich kontinuierlich selbst erneuern. Gergs zeigt Prinzipien für ein Change Management, das den Anforderungen agiler Organisationen Rechnung trägt, indem es eine solche kontinuierliche Selbsterneuerung ermöglicht. Anhand von Fallstudien findet der Autor Indizien für die These, dass kontinuierliche Selbsterneuerung nach völlig anderen Prinzipien funktioniert als die etablierte Praxis der Gestaltung von Veränderungsprozessen.

Miriam Rennung und Anja Göritz setzen sich mit einem spannenden Aspekt von Organisationen auseinander, nämlich mit Ritualen. In ihrer Interviewstudie Die Wirkung organisationaler Rituale untersuchen sie, wie Organisationsmitglieder Rituale wahrnehmen und mithilfe welcher impliziten Theorien sie deren Wirkung erklären. Die Ergebnisse zeigen, dass Rituale aus der Sicht der Teilnehmer/-innen nicht nur die in der Literatur am häufigsten genannten Funktionen (z. B. Sinnstiftung oder Stärkung von Beziehungen), sondern darüber hinaus eine Reihe anderer, unmittelbar für die Arbeitserledigung relevanter Funktionen erfüllen.

Mit zunehmender Vernetztheit von Arbeitsabläufen gewinnt das Thema Feedback an Bedeutung - nicht allein ,top down“ von Führungskräften an ihre Mitarbeitenden, sondern auf allen Ebenen innerhalb der Organisation ebenso wie in der Beziehung zu externen Stakeholdern. Karl Schattenhofer beleuchtet das Thema in seinem Überblicksartikel Feedback in Organisationen; besondere Aufmerksamkeit widmet er dabei dem Problem der Anonymität in Feedbacks sowie „Beraterkunstfehlern“ im Umgang mit Feedback.

In der Rubrik Aktuelles widmen sich Heidi Möller und Alessa Müller einem Phänomen, das in den letzten Jahren 
immer wieder für Aufmerksamkeit gesorgt hat. Der Begriff des Manager-Derailments beschreibt eine Situation, in der Top-Führungskräfte plötzlich ,entgleisen“ und scheitern. In der VUKA-Welt sehen sich Manager immer häufiger mit der Gefahr des Scheiterns konfrontiert - insofern schlägt dieser Beitrag den Bogen zurück zum Heftthema.

Wir wünschen allen Leserinnen und Lesern eine spannende und erkenntnisreiche Lektüre!

Conny Antoni \& Falko von Ameln

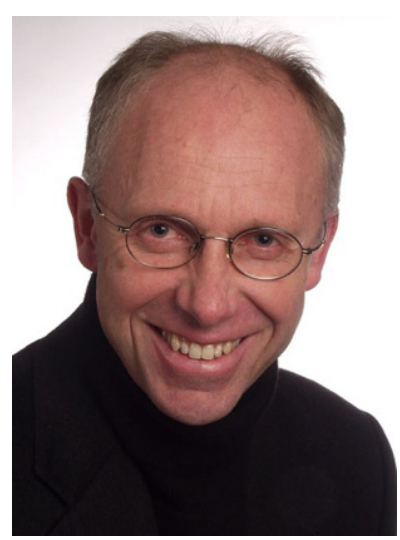

Dr. Conny Herbert Antoni ist Professor für Arbeits-, Betriebsund Organisationspsychologie an der Universität Trier. Seine Arbeitsschwerpunkte liegen in den Bereichen Teamarbeit, Führung, Stress und Work-Life Balance, Reward und Change Management.

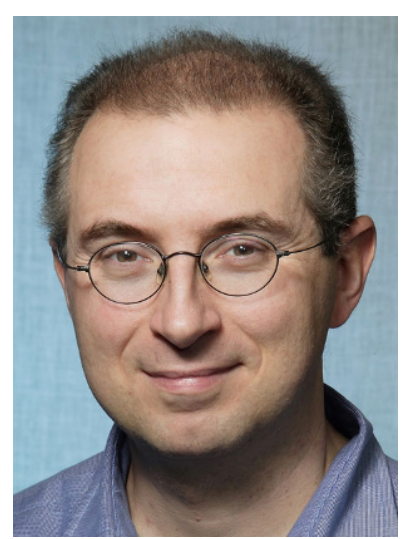

Falko von Ameln Priv.-Doz. Dr. Falko von Ameln ist Organisationsberater und an verschiedenen Universitäten und Instituten in der Ausbildung von Berater/innen engagiert. Habilitation im Fach Beratungswissenschaft, Editor-in-Chief der Zeitschrift „Gruppe. Interaktion. Organisation“. 